Jolanta SocaŁa (Racibórz)

WOJCIECH M. ZAJĄCZKOWSKI (Warszawa)

\title{
LONG TIME EXISTENCE OF REGULAR SOLUTIONS TO 3D NAVIER-STOKES EQUATIONS COUPLED WITH HEAT CONVECTION
}

Abstract. We prove long time existence of regular solutions to the NavierStokes equations coupled with the heat equation. We consider the system in a non-axially symmetric cylinder, with the slip boundary conditions for the Navier-Stokes equations, and the Neumann condition for the heat equation. The long time existence is possible because the derivatives, with respect to the variable along the axis of the cylinder, of the initial velocity, initial temperature and external force are assumed to be sufficiently small in the $L_{2}$ norms. We prove the existence of solutions such that the velocity and temperature belong to $W_{\sigma}^{2,1}(\Omega \times(0, T))$, where $\sigma>5 / 3$. The existence is proved by using the Leray-Schauder fixed point theorem.

1. Introduction. We consider the following problem:

$$
\begin{array}{ll}
v_{, t}+v \cdot \nabla v-\operatorname{div} \mathbb{T}(v, p)=\alpha(\theta) f & \text { in } \Omega^{T}=\Omega \times(0, T), \\
\operatorname{div} v=0 & \text { in } \Omega^{T}, \\
\theta_{, t}+v \cdot \nabla \theta-\varkappa \Delta \theta=0 & \text { in } \Omega^{T}, \\
\bar{n} \cdot \mathbb{D}(v) \cdot \bar{\tau}_{\alpha}=0, \quad \alpha=1,2 & \text { on } S^{T}=S \times(0, T), \\
\bar{n} \cdot \bar{v}=0 & \text { on } S^{T}, \\
\bar{n} \cdot \nabla \theta=0 & \text { on } S^{T}, \\
\left.v\right|_{t=0}=v_{0},\left.\quad \theta\right|_{t=0}=\theta_{0} & \text { in } \Omega,
\end{array}
$$

2010 Mathematics Subject Classification: Primary 35D05; Secondary 35D10, 35K05, 35K20, 35Q30, 76D03, 76D05.

Key words and phrases: Navier-Stokes equations, heat equation, coupled, slip boundary conditions, Neumann condition, long time existence, regular solutions. 
where $\Omega \subset \mathbb{R}^{3}$ is a cylindrical domain, $S=\partial \Omega, v=\left(v_{1}(x, t), v_{2}(x, t), v_{3}(x, t)\right)$ $\in \mathbb{R}^{3}$ is the velocity of the fluid motion, $p=p(x, t) \in \mathbb{R}^{1}$ the pressure, $\theta=\theta(x, t) \in \mathbb{R}_{+}$the temperature, $f=\left(f_{1}(x, t), f_{2}(x, t), f_{3}(x, t)\right) \in \mathbb{R}^{3}$ the external force field, $\bar{n}$ is the unit outward normal vector to the boundary $S$, $\bar{\tau}_{\alpha}, \alpha=1,2$, are tangent vectors to $S$ and the dot denotes the scalar product in $\mathbb{R}^{3}$. We define the stress tensor by

$$
\mathbb{T}(v, p)=\nu \mathbb{D}(v)-p \mathbb{I},
$$

where $\nu$ is the constant viscosity coefficient, $\mathbb{I}$ is the unit matrix and $\mathbb{D}(v)$ is the dilatation tensor of the form

$$
\mathbb{D}(v)=\left\{v_{i, x_{j}}+v_{j, x_{i}}\right\}_{i, j=1,2,3} .
$$

Finally $\varkappa$ is a positive heat conductivity coefficient.

By $x=\left(x_{1}, x_{2}, x_{3}\right)$ we denote the Cartesian coordinates, $\Omega \subset \mathbb{R}^{3}$ is a cylindrical type domain parallel to the $x_{3}$ axis with arbitrary cross section.

We assume that $S=S_{1} \cup S_{2}$, where $S_{1}$ is the part of the boundary which is parallel to the $x_{3}$ axis and $S_{2}$ is perpendicular to that axis. More precisely,

$$
\begin{aligned}
& S_{1}=\left\{x \in \mathbb{R}^{3}: \varphi_{0}\left(x_{1}, x_{2}\right)=c_{*},-b<x_{3}<b\right\}, \\
& S_{2}=\left\{x \in \mathbb{R}^{3}: \varphi_{0}\left(x_{1}, x_{2}\right)<c_{*}, x_{3} \text { equals either }-b \text { or } b\right\},
\end{aligned}
$$

where $b, c_{*}$ are given positive numbers and $\varphi_{0}\left(x_{1}, x_{2}\right)$ describes a sufficiently smooth closed curve in the plane $x_{3}=$ const. We can assume $\bar{\tau}_{1}=\left(\tau_{11}, \tau_{12}, 0\right)$ $\tau_{2}=(0,0,1)$ and $\bar{n}=\left(\tau_{12},-\tau_{11}, 0\right)$ on $S_{1}$.

We assume $\alpha \in C^{2}\left(\mathbb{R}_{+}\right)$and $\Omega^{T}$ satisfies the weak $l$-horn condition, where $l=(2,2,2,1)$ (see [2, Ch. 2, Sect. 8]). The horn condition is an important element of the proofs of the imbedding theorems for anisotropic Sobolev spaces used in this paper. Moreover we assume $\Omega$ is not axially symmetric.

Now we formulate the main result of this paper. Let $g=f_{, x_{3}}, h=v_{, x_{3}}$, $q=p_{, x_{3}}, \vartheta=\theta_{, x_{3}}, \chi=(\operatorname{rot} v)_{3}, F=(\operatorname{rot} f)_{3}$. Assume that $\left\|\theta_{0}\right\|_{L_{\infty}(\Omega)}<\infty$.

Define

$$
a:[0, \infty) \rightarrow[0, \infty), \quad a(x)=\sup \left\{|\alpha(y)|+\left|\alpha^{\prime}(y)\right|+\left|\alpha^{\prime \prime}(y)\right|:|y| \leq x\right\}
$$

and $c_{1}=a\left(\left\|\theta_{0}\right\|_{L_{\infty}}\right)$. Moreover assume that $5 / 3<\sigma<\infty, 5 / 3<\varrho<\infty$, $5 / \varrho-5 / \sigma<1$ and for $t \leq T$ :

1. $c_{1}\|g\|_{L_{2}\left(0, t ; L_{6 / 5}(\Omega)\right)}+c_{1} c_{0}\|f\|_{L_{\infty}\left(0, t ; L_{3}(\Omega)\right)}+c_{1}\|F\|_{L_{2}\left(0, t ; L_{6 / 5}(\Omega)\right)}$

$+c_{1}\left\|f_{3}\right\|_{L_{2}\left(0, t ; L_{4 / 3}\left(S_{2}\right)\right)}+\left\|h_{0}\right\|_{L_{2}(\Omega)}+\left\|\vartheta_{0}\right\|_{L_{2}(\Omega)}+\left\|\chi_{0}\right\|_{L_{2}(\Omega)}$

$+c_{0}^{2}\left(c_{1}\|f\|_{L_{2}\left(0, t ; L_{6 / 5}(\Omega)\right)}+\left\|v_{0}\right\|_{L_{2}(\Omega)}\right)+\psi\left(c_{0}\right) \leq k_{1}<\infty$,

2. $\|f\|_{L_{2}\left(0, t ; L_{3}(\Omega)\right)} \leq k_{2}<\infty$,

3. $\|f\|_{L_{2}\left(\Omega^{t}\right)}+\left\|v_{0}\right\|_{H^{1}(\Omega)} \leq k_{3}<\infty$,

4. $c_{1}\|f\|_{L_{\infty}\left(\Omega^{t}\right)} e^{c c_{1}^{2} k_{2}^{2}} k_{1}+c_{1}\|g\|_{L_{\sigma}\left(\Omega^{t}\right)}+\left\|\vartheta_{0}\right\|_{W_{\sigma}^{2-2 / \sigma}(\Omega)}+\left\|h_{0}\right\|_{W_{\sigma}^{2-2 / \sigma}(\Omega)} \leq$ $k_{4}<\infty$, 
5. $c_{1}\|g\|_{L_{2}\left(0, t ; L_{6 / 5}(\Omega)\right)}+c_{1}\left\|f_{3}\right\|_{L_{2}\left(0, t ; L_{4 / 3}\left(S_{2}\right)\right)}+\left\|h_{0}\right\|_{L_{2}(\Omega)}+\left\|\vartheta_{0}\right\|_{L_{2}(\Omega)} \leq$ $d<\infty$,

6. $c_{1}\|f\|_{L_{\sigma}\left(\Omega^{t}\right)}+\left\|v_{0}\right\|_{W_{\varrho}^{2-2 / \varrho}(\Omega)}+\left\|\theta_{0}\right\|_{W_{\varrho}^{2-2 / \varrho}(\Omega)} \leq k_{5}<\infty$,

where $c_{0}$ is the constant from Lemma $2.4, \psi\left(c_{0}\right)$ is an increasing function (see Lemma 3.3 in [8]) and $k_{1}, \ldots, k_{5}$ are given constants. Assume

$$
f \in L_{\sigma}\left(\Omega^{T}\right), \quad g \in L_{\sigma}\left(\Omega^{T}\right), \quad \vartheta_{0} \in W_{\sigma}^{2-2 / \sigma}(\Omega) .
$$

TheOREM 1.1. Let the above assumptions hold. Assume that $d$ is sufficiently small (see [5, Main Theorem]). Then there exists a strong solution $(v, p, \theta)$ to $(1.1)$ such that $v, \theta \in W_{\varrho}^{2,1}\left(\Omega^{T}\right), \nabla p \in L_{\varphi}\left(\Omega^{T}\right), h, \vartheta \in W_{\sigma}^{2,1}\left(\Omega^{T}\right)$, $\nabla q \in L_{\sigma}\left(\Omega^{T}\right)$.

The result follows by applying the methods developed in [6] to the more complicated system (1.1). However, the proof of existence is now much clearer than in [6], because the mapping $\phi$ is constructed in a simpler way. This, however, needs more regularity. Therefore in this paper we prove the existence of much more regular solutions than in [6].

Problem (1.1) in the case of inflow-outflow was generalized in [3, 4]. Papers [3, 4] base on [13, where the inflow-outflow problem was considered for the Navier-Stokes equations in a cylindrical pipe.

2. Preliminaries. The notation used in this paper is the same as in 8 , Sect. 2.1]. The definition of weak solution is introduced in [8, Sect. 2.2]. Now we recall some important results employed in this paper.

Lemma 2.1 (see [12], Korn inequality). Assume that

$$
E_{\Omega}(v)=\|\mathbb{D}(v)\|_{L_{2}(\Omega)}^{2}<\infty,\left.\quad v \cdot \bar{n}\right|_{S}=0, \quad \operatorname{div} v=0 .
$$

If $\Omega$ is not axially symmetric there exists a constant $c_{1}$ independent of $v$ such that

$$
\|v\|_{H^{1}(\Omega)}^{2} \leq c E_{\Omega}(v) .
$$

If $\Omega$ is axially symmetric, $\eta=\left(-x_{2}, x_{1}, 0\right), \alpha=\int_{\Omega} v \cdot \eta d x$, then there exists a constant $c$ independent of $v$ such that

$$
\|v\|_{H^{1}(\Omega)}^{2} \leq c\left(E_{\Omega}(v)+|\alpha|^{2}\right) .
$$

Let us consider the problem

$$
\begin{array}{ll}
h_{, t}-\operatorname{div} \mathbb{T}(h, q)=f & \text { in } \Omega^{T}, \\
\operatorname{div} h=0 & \text { in } \Omega^{T}, \\
\bar{n} \cdot h=0, \quad \bar{n} \cdot \mathbb{D}(h) \cdot \bar{\tau}_{\alpha}=0, \quad \alpha=1,2, & \text { on } S_{1}^{T}, \\
h_{i}=0, \quad i=1,2, \quad h_{3, x_{3}}=0 & \text { on } S_{2}^{T}, \\
\left.h\right|_{t=0}=h_{0} & \text { in } \Omega .
\end{array}
$$


Theorem 2.2. Let $f \in L_{p}\left(\Omega^{T}\right), h(0) \in W_{p}^{2-2 / p}(\Omega), S_{i} \in C^{2}, i=1,2$, $1<p<\infty$. Then there exists a solution to problem (2.4) such that $h \in$ $W_{p}^{2,1}\left(\Omega^{T}\right), \nabla q \in L_{p}\left(\Omega^{T}\right)$ and there exists a constant $c$ depending on $S$ and $p$ such that

$$
\|h\|_{W_{p}^{2,1}\left(\Omega^{T}\right)}+\|\nabla q\|_{L_{p}\left(\Omega^{T}\right)} \leq c\left(\|f\|_{L_{p}\left(\Omega^{T}\right)}+\left\|h_{0}\right\|_{W_{p}^{2-2 / p}(\Omega)}\right) .
$$

The proof follows from considerations in [5, Ch. 4].

Let us consider the problem

$$
\begin{array}{ll}
v_{, t}-\operatorname{div} \mathbb{T}(v, q)=f & \text { in } \Omega^{T}, \\
\operatorname{div} v=0 & \text { in } \Omega^{T}, \\
\bar{n} \cdot v=0, \quad \bar{n} \cdot \mathbb{D}(v) \cdot \bar{\tau}_{\alpha}=0, \quad \alpha=1,2, & \text { on } S^{T}, \\
\left.v\right|_{t=0}=v_{0} & \text { in } \Omega .
\end{array}
$$

TheOREM 2.3. Let $f \in L_{p}\left(\Omega^{T}\right), v_{0} \in W_{p}^{2-2 / p}(\Omega), S_{i} \in C^{2}, i=1,2$, $1<p<\infty$. Then there exists a solution to problem (2.6) such that $v \in$ $W_{p}^{2,1}\left(\Omega^{T}\right), \nabla p \in L_{p}\left(\Omega^{T}\right)$ and there exists a constant $c$ depending on $S$ and p such that

$$
\|v\|_{W_{p}^{2,1}\left(\Omega^{T}\right)}+\|\nabla q\|_{L_{p}\left(\Omega^{T}\right)} \leq c\left(\|f\|_{L_{p}\left(\Omega^{T}\right)}+\left\|v_{0}\right\|_{W_{p}^{2-2 / p}(\Omega)}\right) .
$$

The proof is similar to the proof from [1].

Lemma 2.4 (see [8, Lemma 2.3]). Assume that $v_{0} \in L_{2}(\Omega), \theta_{0} \in L_{\infty}(\Omega)$, $f \in L_{2}\left(0, T ; L_{6 / 5}(\Omega)\right), T<\infty$. Assume that $\Omega$ is not axially symmetric. Assume that there exist constants $\theta_{*}, \theta^{*}$ such that $\theta_{*}<\theta^{*}$ and

$$
\theta_{*} \leq \theta_{0}(x) \leq \theta^{*}, \quad x \in \Omega .
$$

Then there exists a weak solution to problem (1.1) such that $(v, \theta) \in V_{2}^{0}\left(\Omega^{T}\right)$ $\times V_{2}^{0}\left(\Omega^{T}\right), \theta \in L_{\infty}\left(\Omega^{T}\right)$ and

$$
\theta_{*} \leq \theta(x, t) \leq \theta^{*}, \quad(x, t) \in \Omega^{T},
$$

and there exist positive constants $c, c_{0}$ independent of $v$ and $\theta$ such that

$$
\begin{gathered}
\|v\|_{V_{2}^{0}\left(\Omega^{T}\right)} \leq c\left(a\left(\left\|\theta_{0}\right\|_{L_{\infty}(\Omega)}\right)\|f\|_{L_{2}\left(0, T ; L_{6 / 5}(\Omega)\right)}+\left\|v_{0}\right\|_{L_{2}(\Omega)}\right) \leq c_{0}, \\
\|\theta\|_{V_{2}^{0}\left(\Omega^{T}\right)} \leq c\left\|\theta_{0}\right\|_{L_{2}(\Omega)} \leq c_{0} .
\end{gathered}
$$

REMARK 2.5. If $\theta(0) \geq 0$, then $\theta(t) \geq 0$ for $t \geq 0$.

3. Existence. For $\xi, \eta, \sigma, \varrho \geq 1$ define

$$
\begin{aligned}
\|(v, \theta)\|_{\mathcal{M}\left(\Omega^{T}\right)}= & \|v\|_{L_{\infty}\left(0, T ; W_{\eta}^{1}(\Omega)\right)}+\|\theta\|_{L_{\infty}\left(0, T ; W_{\eta}^{1}(\Omega)\right)} \\
& +\left\|v_{x_{3}}\right\|_{L_{\infty}\left(0, T ; W_{\xi}^{1}(\Omega)\right)}+\left\|\theta_{, x_{3}}\right\|_{L_{\infty}\left(0, T ; W_{\xi}^{1}(\Omega)\right)},
\end{aligned}
$$




$$
\begin{aligned}
\mathcal{M}\left(\Omega^{T}\right)= & \left\{(v, \theta):\|(v, \theta)\|_{\mathcal{M}\left(\Omega^{T}\right)}<\infty\right\} \\
\|(v, \theta)\|_{\mathcal{N}\left(\Omega^{T}\right)}= & \|v\|_{W_{\varrho}^{2,1}\left(\Omega^{T}\right)}+\|\theta\|_{W_{\varrho}^{2,1}\left(\Omega^{T}\right)} \\
& +\left\|v_{, x_{3}}\right\|_{W_{\sigma}^{2,1}\left(\Omega^{T}\right)}+\left\|\theta_{, x_{3}}\right\|_{W_{\sigma}^{2,1}\left(\Omega^{T}\right)}, \\
\mathcal{N}\left(\Omega^{T}\right)= & \left\{(v, \theta):\|(v, \theta)\|_{\mathcal{N}\left(\Omega^{T}\right)}<\infty\right\} .
\end{aligned}
$$

LEMMA 3.1.

1. $\left(\mathcal{M}\left(\Omega^{T}\right),\|\|_{\mathcal{M}\left(\Omega^{T}\right)}\right)$ is a Banach space.

2. $\left(\mathcal{N}\left(\Omega^{T}\right),\|\|_{\mathcal{N}\left(\Omega^{T}\right)}\right)$ is a Banach space.

3. $\|u\|_{\mathcal{M}\left(\Omega^{T}\right)} \leq c\|u\|_{\mathcal{N}\left(\Omega^{T}\right)}$ for $u \in \mathcal{N}\left(\Omega^{T}\right)$ and the imbedding $\mathcal{N}\left(\Omega^{T}\right) \subset$ $\mathcal{M}\left(\Omega^{T}\right)$ is compact for $\varrho<\eta, 5 / \varrho-3 / \eta<1, \sigma<\xi, 5 / \sigma-3 / \xi<1$.

Let us consider the problems

$$
\begin{aligned}
& v_{t}-\operatorname{div} \mathbb{T}(v, p)=-\lambda[\tilde{v} \cdot \nabla \tilde{v}+\alpha(\tilde{\theta}) f], \\
& \operatorname{div} v=0, \\
& \left.v \cdot \bar{n}\right|_{S}=0,\left.\quad \bar{n} \cdot \mathbb{D}(v) \cdot \bar{\tau}_{\alpha}\right|_{S}=0, \quad \alpha=1,2, \\
& \left.v\right|_{t=0}=v_{0}
\end{aligned}
$$

and

$$
\begin{aligned}
& \theta_{t}-\varkappa \Delta \theta=-\lambda \tilde{v} \cdot \nabla \tilde{\theta}, \\
& \left.\bar{n} \cdot \nabla \theta\right|_{S}=0, \\
& \left.\theta\right|_{t=0}=\theta_{0}
\end{aligned}
$$

where $\lambda \in[0,1]$ is a parameter and $\tilde{v}, \tilde{\theta}$ are treated as given functions. We will assume that $\alpha \in C^{2}(\mathbb{R})$.

LEMMA 3.2. Assume that

$$
\begin{aligned}
& (\tilde{v}, \tilde{\theta}) \in \mathcal{M}\left(\Omega^{T}\right), \quad 3<\eta<\infty, \\
& f \in L_{\varrho}\left(\Omega^{T}\right), \quad 1<\varrho<\infty, \\
& v_{0} \in W_{\varrho}^{2-2 / \varrho}(\Omega), \\
& S_{i} \in C^{2}, \quad i=1,2, \quad 5 / \varrho-3 / \eta<1, \quad \varrho<\eta .
\end{aligned}
$$

Then there exists a unique solution to problem (3.1) such that

$$
v \in W_{\varrho}^{2,1}\left(\Omega^{T}\right) \subset L_{\infty}\left(0, T ; W_{\eta}^{1}(\Omega)\right)
$$

and

$$
\begin{aligned}
& \|v\|_{L_{\infty}\left(0, T ; W_{\eta}^{1}(\Omega)\right)} \leq c\|v\|_{W_{\varrho}^{2,1}\left(\Omega^{T}\right)} \\
& \quad \leq c\left(\lambda\|(\tilde{v}, \tilde{\theta})\|_{\mathcal{M}\left(\Omega^{T}\right)}^{2}+\lambda a\left(c\|(\tilde{v}, \tilde{\theta})\|_{\mathcal{M}\left(\Omega^{T}\right)}\right)\|f\|_{L_{\varrho}\left(\Omega^{T}\right)}+\left\|v_{0}\right\|_{W_{\varrho}^{2-2 / \varrho}(\Omega)}\right) .
\end{aligned}
$$


Proof. We have

$$
\begin{aligned}
\|\tilde{v} \cdot \nabla \tilde{v}\|_{L_{\varrho}\left(\Omega^{T}\right)} & \leq c\|\tilde{v}\|_{L_{\infty}\left(\Omega^{T}\right)}\|\nabla \tilde{v}\|_{L_{\eta}\left(\Omega^{T}\right)} \leq c\|\tilde{v}\|_{L_{\infty}\left(0, T ; W_{\eta}^{1}(\Omega)\right)}^{2} \\
& \leq c\|(\tilde{v}, \tilde{\theta})\|_{\mathcal{M}\left(\Omega^{T}\right)}^{2}
\end{aligned}
$$

and

$$
\begin{aligned}
\|\alpha(\tilde{\theta}) f\|_{L_{\varrho}\left(\Omega^{T}\right)} & \leq a\left(c\|\tilde{\theta}\|_{L_{\infty}\left(0, T ; W_{\eta}^{1}(\Omega)\right)}\right)\|f\|_{L_{\varrho}\left(\Omega^{T}\right)} \\
& \leq a\left(c\|(\tilde{v}, \tilde{\theta})\|_{\mathcal{M}\left(\Omega^{T}\right)}\right)\|f\|_{L_{\varrho}\left(\Omega^{T}\right)} .
\end{aligned}
$$

By Theorem 2.2 the proof is complete.

LEMMA 3.3. Assume that

$$
\begin{aligned}
& 3<\eta<\infty, \quad 1<\varrho<\infty, \quad \varrho<\eta, \quad 5 / \varrho-3 / \eta<1, \\
& (\tilde{v}, \tilde{\theta}) \in \mathcal{M}\left(\Omega^{T}\right), \quad \theta_{0} \in W_{\varrho}^{2-2} \varrho(\Omega) .
\end{aligned}
$$

Then there exists a unique solution to problem (3.2) such that

$$
\theta \in W_{\varrho}^{2,1}\left(\Omega^{T}\right) \subset L_{\infty}\left(0, T ; W_{\eta}^{1}(\Omega)\right)
$$

and

$$
\|\theta\|_{L_{\infty}\left(0, T ; W_{\eta}^{1}(\Omega)\right)} \leq c\|\theta\|_{W_{\varrho}^{2,1}\left(\Omega^{T}\right)} \leq c\left(\lambda\|(\tilde{v}, \tilde{\theta})\|_{\mathcal{M}\left(\Omega^{T}\right)}^{2}+\left\|\theta_{0}\right\|_{W_{\varrho}^{2-2 / \varrho}(\Omega)}\right) .
$$

Proof. We have

$$
\begin{aligned}
\|\tilde{v} \cdot \nabla \tilde{\theta}\|_{L_{\varrho}\left(\Omega^{T}\right)} & \leq\|\tilde{v}\|_{L_{\infty}\left(\Omega^{T}\right)}\|\nabla \tilde{\theta}\|_{L_{\eta}\left(\Omega^{T}\right)} \\
& \leq c\|\tilde{v}\|_{L_{\infty}\left(0, T ; W_{\eta}^{1}(\Omega)\right)}\|\tilde{\theta}\|_{L_{\infty}\left(0, T ; W_{\eta}^{1}(\Omega)\right)} \leq c\|(\tilde{v}, \tilde{\theta})\|_{\mathcal{M}\left(\Omega^{T}\right)}^{2}
\end{aligned}
$$

Then we argue as for Theorem 9.1 in [5, Ch. 4, Sect. 9] (see also [9, Theorem 17]).

\section{LEMMA 3.4. Let}

$$
\begin{aligned}
& (\tilde{v}, \tilde{\theta}) \in \mathcal{M}\left(\Omega^{T}\right), \quad 3<\xi<\infty, \quad 3<\eta<\infty, \\
& f \in L_{\sigma}\left(\Omega^{T}\right), \quad g \in L_{\sigma}\left(\Omega^{T}\right), \quad 1<\sigma<\infty \quad\left(\text { where } g=f_{x_{3}}\right), \\
& \sigma<\eta, \quad S_{i} \in C^{2}, \quad i=1,2, \quad \sigma<\xi, \quad 5 / \sigma-3 / \xi<1 .
\end{aligned}
$$

Let $v, p$ be a unique solution to problem (3.1). Let $h=v_{, x_{3}}, q=p_{, x_{3}}$. Assume $h_{0} \in W_{\sigma}^{2-2 / \sigma}(\Omega)$. Then

$$
h \in W_{\sigma}^{2,1}\left(\Omega^{T}\right) \subset L_{\infty}\left(0, T ; W_{\xi}^{1}(\Omega)\right)
$$

and

$$
\begin{aligned}
\|h\|_{L_{\infty}\left(0, T ; W_{\xi}^{1}(\Omega)\right)} \leq c\|h\|_{W_{\sigma}^{2,1}\left(\Omega^{T}\right)} & \\
\leq & c\left(\lambda\|(\tilde{v}, \tilde{\theta})\|_{\mathcal{M}\left(\Omega^{T}\right)}^{2}+\lambda a\left(c\|(\tilde{v}, \tilde{\theta})\|_{\mathcal{M}\left(\Omega^{T}\right)}\right)\|(\tilde{v}, \tilde{\theta})\|_{\mathcal{M}\left(\Omega^{T}\right)}\|f\|_{L_{\sigma}\left(\Omega^{T}\right)}\right. \\
& \left.+\lambda a\left(c\|(\tilde{v}, \tilde{\theta})\|_{\mathcal{M}\left(\Omega^{T}\right)}\right)\|g\|_{L_{\sigma}\left(\Omega^{T}\right)}+\left\|h_{0}\right\|_{W_{\sigma}^{2-2 / \sigma}(\Omega)}\right) .
\end{aligned}
$$


Proof. The function $h$ is a solution of the following problem:

$$
\begin{array}{ll}
h_{, t}-\operatorname{div} \mathbb{T}(h, q)=\lambda\left[-\tilde{v} \cdot \nabla \tilde{h}-\tilde{h} \cdot \nabla \tilde{v}+\alpha_{\theta}(\tilde{\theta}) \tilde{v} f+\alpha(\tilde{\theta}) g\right] & \text { in } \Omega^{T}, \\
\operatorname{div} h=0 & \text { in } \Omega^{T}, \\
\bar{n} \cdot h=0, \quad \bar{n} \cdot \mathbb{D}(h) \cdot \bar{\tau}_{\alpha}=0, \quad \alpha=1,2, & \text { on } S_{1}^{T}, \\
h_{i}=0, \quad i=1,2, \quad h_{3, x_{3}}=0 & \text { on } S_{2}^{T}, \\
\left.h\right|_{t=0}=h_{0} & \text { in } \Omega,
\end{array}
$$

where $\tilde{h}=\tilde{v}_{x_{3}}, \tilde{\vartheta}=\tilde{\theta}_{, x_{3}}$. We have

$$
\begin{aligned}
\|\tilde{v} \cdot \nabla \tilde{h}\|_{L_{\sigma}\left(\Omega^{T}\right)} & \leq c\|\tilde{v}\|_{L_{\infty}\left(\Omega^{T}\right)}\|\nabla \tilde{h}\|_{L_{\xi}\left(\Omega^{T}\right)} \\
& \leq c\|\tilde{v}\|_{L_{\infty}\left(0, T ; W_{\eta}^{1}(\Omega)\right)}\|\tilde{h}\|_{L_{\infty}\left(0, T ; W_{\xi}^{1}(\Omega)\right)} \leq c\|(\tilde{v}, \tilde{\theta})\|_{\mathcal{M}\left(\Omega^{T}\right)}^{2}
\end{aligned}
$$

and

$$
\begin{aligned}
\|\tilde{h} \cdot \nabla \tilde{v}\|_{L_{\sigma}\left(\Omega^{T}\right)} & \leq c\|\tilde{h}\|_{L_{\infty}\left(\Omega^{T}\right)}\|\nabla \tilde{v}\|_{L_{\eta}\left(\Omega^{T}\right)} \\
& \leq c\|\tilde{h}\|_{L_{\infty}\left(0, T ; W_{\xi}^{1}(\Omega)\right)}\|\tilde{v}\|_{L_{\infty}\left(0, T ; W_{\eta}^{1}(\Omega)\right)} \leq c\|(\tilde{v}, \tilde{\theta})\|_{\mathcal{M}\left(\Omega^{T}\right)}^{2} .
\end{aligned}
$$

Next

$$
\begin{aligned}
\left\|\alpha_{\theta}(\tilde{\theta}) \tilde{\vartheta} f\right\|_{L_{\sigma}\left(\Omega^{T}\right)} & \leq c a\left(c\|\tilde{\theta}\|_{L_{\infty}\left(0, T ; W_{\eta}^{1}(\Omega)\right)}\right)\|\tilde{\vartheta}\|_{L_{\infty}\left(0, T ; W_{\xi}^{1}(\Omega)\right)}\|f\|_{L_{\sigma}\left(\Omega^{T}\right)} \\
& \leq c a\left(c\|(\tilde{v}, \tilde{\theta})\|_{\mathcal{M}\left(\Omega^{T}\right)}\right)\|(\tilde{v}, \tilde{\theta})\|_{\mathcal{M}(\Omega)}\|f\|_{L_{\sigma}\left(\Omega^{T}\right)}
\end{aligned}
$$

and

$$
\begin{aligned}
\|\alpha(\tilde{\theta}) g\|_{L_{\sigma}\left(\Omega^{T}\right)} & \leq a\left(c\|\tilde{\theta}\|_{L_{\infty}\left(0, T ; W_{\eta}^{1}(\Omega)\right)}\right)\|g\|_{L_{\sigma}\left(\Omega^{T}\right)} \\
& \leq a\left(c\|(\tilde{v}, \tilde{\theta})\|_{\mathcal{M}\left(\Omega^{T}\right)}\right)\|g\|_{L_{\sigma}\left(\Omega^{T}\right)} .
\end{aligned}
$$

By Theorem 2.1 the proof is complete.

Lemma 3.5. Assume that $3<\eta<\infty, 1<\sigma<\infty, \sigma<\eta, 5 / \sigma-3 / \xi<1$, $3<\xi<\infty, \sigma<\xi$, $(\tilde{v}, \tilde{\theta}) \in \mathcal{M}\left(\Omega^{T}\right)$. Let $\theta$ be a unique solution to problem (3.2). Let $\vartheta=\theta_{, x_{3}}$. Assume that $\vartheta(0) \in W_{\sigma}^{2-2 / \sigma}(\Omega)$. Then

$$
\vartheta \in W_{\sigma}^{2,1}\left(\Omega^{T}\right) \subset L_{\infty}\left(0, T ; W_{\xi}^{1}(\Omega)\right)
$$

and

$$
\|\vartheta\|_{L_{\infty}\left(0, T ; W_{\xi}^{1}(\Omega)\right)} \leq c\|\vartheta\|_{W_{\sigma}^{2,1}\left(\Omega^{T}\right)} \leq c\left(\lambda\|(\tilde{v}, \tilde{\theta})\|_{\mathcal{M}\left(\Omega^{T}\right)}^{2}+\|\vartheta(0)\|_{W_{\sigma}^{2-2 / \sigma}(\Omega)}\right) .
$$

Proof. The function $\vartheta$ is a solution of the problem

$$
\begin{array}{ll}
\vartheta_{, t}-\varkappa \Delta \vartheta=-\lambda[\tilde{h} \cdot \nabla \tilde{\theta}+\tilde{v} \cdot \nabla \tilde{\vartheta}] & \text { in } \Omega^{T}, \\
\bar{n} \cdot \nabla \vartheta=0 & \text { on } S_{1}^{T}, \\
\vartheta=0 & \text { on } S_{2}^{T}, \\
\left.\vartheta\right|_{t=0}=\vartheta_{0} & \text { in } \Omega,
\end{array}
$$


where $\tilde{\vartheta}=\tilde{\theta}_{, x_{3}}$. We have

$$
\begin{aligned}
\|\tilde{h} \cdot \nabla \tilde{\theta}\|_{L_{\sigma}\left(\Omega^{T}\right)} & \leq\|\tilde{h}\|_{L_{\infty}\left(\Omega^{T}\right)}\|\nabla \tilde{\theta}\|_{L_{\eta}\left(\Omega^{T}\right)} \leq c\|\tilde{h}\|_{L_{\infty}\left(0, T ; W_{\xi}^{1}(\Omega)\right)}\|\tilde{\theta}\|_{L_{\infty}\left(0, T ; W_{\eta}^{1}(\Omega)\right)} \\
& \leq c\|(\tilde{v}, \tilde{\theta})\|_{\mathcal{M}\left(\Omega^{T}\right)}^{2}
\end{aligned}
$$

and

$$
\begin{aligned}
\|\tilde{v} \cdot \nabla \tilde{\vartheta}\|_{L_{\sigma}\left(\Omega^{T}\right)} & \leq\|\tilde{v}\|_{L_{\infty}\left(\Omega^{T}\right)}\|\nabla \tilde{\vartheta}\|_{L_{\xi}\left(\Omega^{T}\right)} \leq c\|\tilde{v}\|_{L_{\infty}\left(0, T ; W_{\eta}^{1}(\Omega)\right)}\|\tilde{\vartheta}\|_{L_{\infty}\left(0, T ; W_{\xi}^{1}(\Omega)\right)} \\
& \leq c\|(\tilde{v}, \tilde{\theta})\|_{\mathcal{M}\left(\Omega^{T}\right)}^{2} .
\end{aligned}
$$

Then we argue as for Theorem 9.1 in [5, Ch. 4, Sect. 9] (see also [9, Theorem 17]).

From Lemmas 3.1-3.5 it follows that if $(\tilde{v}, \tilde{\theta}) \in \mathcal{M}\left(\Omega^{T}\right)$, then there exists a unique solution $(v, \theta)$ to problems (3.1)-(3.2) such that $(v, \theta) \in \mathcal{M}\left(\Omega^{T}\right)$.

To prove the existence of solutions to problem (1.1) we apply the LeraySchauder fixed point theorem (see [7, 10, 11]). Therefore we introduce the mapping $\phi:[0,1] \times \mathcal{M}\left(\Omega^{T}\right) \rightarrow \mathcal{M}\left(\Omega^{T}\right),(\lambda, \tilde{v}, \tilde{\theta}) \mapsto \phi(\lambda, \tilde{v}, \tilde{\theta})=(v, \theta)$, where $(v, \theta)$ is a solution to problems (3.1)-(3.2).

For $\lambda=0$ we have the existence of a unique solution. For $\lambda=1$ every fixed point is a solution to problem (1.1).

LEMmA 3.6. Let the assumptions of Lemmas 3.2-3.5 be satisfied. Then the mappings $\phi(\lambda, \cdot): \mathcal{M}\left(\Omega^{T}\right) \rightarrow \mathcal{M}\left(\Omega^{T}\right), \lambda \in[0,1]$, are completely continuous.

Proof. By Lemmas 3.1-3.5 the mappings $\phi(\lambda, \cdot), \lambda \in[0,1]$, are compact. It follows that bounded sets in $\mathcal{M}\left(\Omega^{T}\right)$ are transformed into bounded sets in $\mathcal{M}\left(\Omega^{T}\right)$. Let $\left(\tilde{v}_{i}, \tilde{\theta}_{i}\right) \in \mathcal{M}\left(\Omega^{T}\right), i=1,2$, be two given elements. Then $\left(v_{i}, \theta_{i}\right)$, $i=1,2$, are solutions to the problems

$$
\begin{aligned}
& v_{i t}-\operatorname{div} \mathbb{T}\left(v_{i}, p_{i}\right)=-\lambda\left(\tilde{v}_{i} \cdot \nabla \tilde{v}_{i}+\alpha\left(\tilde{\theta}_{i}\right) f\right), \\
& \operatorname{div} v_{i}=0, \\
& \left.\bar{n} \cdot \mathbb{D}\left(v_{i}\right) \cdot \bar{\tau}\right|_{S}=0,\left.\quad \bar{n} \cdot v_{i}\right|_{S}=0, \\
& \left.v_{i}\right|_{t=0}=v_{0}, \quad i=1,2,
\end{aligned}
$$

and

$$
\begin{aligned}
& \theta_{i t}-\varkappa \Delta \theta_{i}=-\lambda \tilde{v}_{i} \cdot \nabla \tilde{\theta}_{i}, \\
& \left.\bar{n} \cdot \nabla \theta_{i}\right|_{S}=0, \\
& \left.\theta_{i}\right|_{t=0}=\theta_{0}, \quad i=1,2 .
\end{aligned}
$$

To show continuity we introduce the differences

$$
V=v_{1}-v_{2}, \quad P=p_{1}-p_{2}, \quad \mathcal{T}=\theta_{1}-\theta_{2},
$$


which are solutions to the problems

$$
\begin{aligned}
& V_{t}-\operatorname{div} \mathbb{T}(V, P)=-\lambda\left[\tilde{V} \cdot \nabla \tilde{v}_{1}+\tilde{v}_{2} \cdot \nabla \tilde{V}+\left(\alpha\left(\tilde{\theta}_{1}\right)-\alpha\left(\tilde{\theta}_{2}\right)\right) f\right] \\
& \operatorname{div} V=0, \\
& \left.V \cdot \bar{n}\right|_{S}=0,\left.\quad \bar{n} \cdot \mathbb{D}(V) \cdot \bar{\tau}\right|_{S}=0 \\
& \left.V\right|_{t=0}=0,
\end{aligned}
$$

and

$$
\begin{aligned}
& \mathcal{T}_{t}-\varkappa \Delta \mathcal{T}=-\lambda\left[\tilde{V} \cdot \nabla \tilde{\theta}_{1}+\tilde{v}_{2} \cdot \nabla \tilde{\mathcal{T}}\right] \\
& \left.\bar{n} \cdot \nabla \mathcal{T}\right|_{S}=0 \\
& \left.\mathcal{T}\right|_{t=0}=0
\end{aligned}
$$

where $\tilde{V}=\tilde{v}_{1}-\tilde{v}_{2}, \tilde{\mathcal{T}}=\tilde{\theta}_{1}-\tilde{\theta}_{2}$. In view of [6] and [10, 11] we have

$$
\begin{aligned}
& \|V\|_{W_{\varrho}^{2,1}\left(\Omega^{T}\right)}+\|\mathcal{T}\|_{W_{\varrho}^{2,1}\left(\Omega^{T}\right)} \\
& \leq c\left[\|\tilde{V}\|_{L_{\infty}\left(\Omega^{T}\right)}\left\|\nabla \tilde{v}_{1}\right\|_{L_{\varrho}\left(\Omega^{T}\right)}+\left\|\tilde{v}_{2}\right\|_{L_{\infty}\left(\Omega^{T}\right)}\|\nabla \tilde{V}\|_{L_{\varrho}\left(\Omega^{T}\right)}\right. \\
& \quad+\operatorname{ca}\left(\max \left\{\left\|\tilde{\theta}_{1}\right\|_{L_{\infty}\left(\Omega^{T}\right)},\left\|\tilde{\theta}_{2}\right\|_{L_{\infty}\left(\Omega^{T}\right)}\right\}\right)\|\tilde{\mathcal{T}}\|_{L_{\infty}\left(\Omega^{T}\right)}\|f\|_{L_{\varrho}\left(\Omega^{T}\right)} \\
& \left.\quad+\left\|\tilde{v}_{2}\right\|_{L_{\infty}\left(\Omega^{T}\right)}\|\nabla \tilde{\mathcal{T}}\|_{L_{\varrho}\left(\Omega^{T}\right)}+\|\tilde{V}\|_{L_{\infty}\left(\Omega^{T}\right)}\left\|\nabla \tilde{\theta}_{1}\right\|_{L_{\varrho}\left(\Omega^{T}\right)}\right] \\
& \leq c\left(\|\tilde{V}\|_{\mathcal{M}\left(\Omega^{T}\right)}+\|\tilde{\mathcal{T}}\|_{\mathcal{M}\left(\Omega^{T}\right)}\right) .
\end{aligned}
$$

Let $h_{i}=v_{i, x_{3}}, q_{i}=p_{i, x_{3}}, \vartheta_{i}=\theta_{i, x_{3}}, \tilde{h}_{i}=\tilde{v}_{i, x_{3}}, \tilde{\vartheta}_{i}=\tilde{\theta}_{i, x_{3}}$. The functions $h_{i}, \vartheta_{i}, i=1,2$, are solutions to the following problems:

$$
\begin{array}{ll}
h_{i, t}-\operatorname{div} \mathbb{T}\left(h_{i}, q_{i}\right)=-\lambda\left[\tilde{h}_{i} \cdot \nabla \tilde{v}_{i}+\tilde{v}_{i} \cdot \nabla \tilde{h}_{i}+\alpha_{\theta}\left(\tilde{\theta}_{i}\right) \tilde{\vartheta}_{i} f+\alpha\left(\tilde{\theta}_{i}\right) g\right] & \text { in } \Omega^{T}, \\
\operatorname{div} h_{i}=0 & \text { in } \Omega^{T}, \\
\bar{n} \cdot h_{i}=0, \quad \bar{n} \cdot \mathbb{D}(h) \cdot \bar{\tau}_{\alpha}, \quad \alpha=1,2, i=1,2, & \text { on } S_{1}^{T}, \\
h_{i j}=0, \quad i=1,2, \quad j=1,2, & \text { on } S_{2}^{T}, \\
h_{i 3, x_{3}}=0, \quad i=1,2, & \text { on } S_{2}^{T}, \\
\left.h_{i}\right|_{t=0}=h_{0} & \text { in } \Omega
\end{array}
$$

and

$$
\begin{array}{ll}
\vartheta_{i, t}-\varkappa \Delta \vartheta_{i}=-\lambda\left[\tilde{h}_{i} \cdot \nabla \tilde{\theta}_{i}+\tilde{v}_{i} \cdot \nabla \tilde{\vartheta}_{i}\right] & \text { in } \Omega^{T}, \\
\bar{n} \cdot \nabla \vartheta_{i}=0 & \text { on } S_{1}^{T}, \\
\vartheta_{i}=0 & \text { on } S_{2}^{T}, \\
\left.\vartheta_{i}\right|_{t=0}=\vartheta_{0} & \text { in } \Omega .
\end{array}
$$

We introduce the differences

$$
H=h_{1}-h_{2}, \quad Q=q_{1}-q_{2}, \quad R=\vartheta_{1}-\vartheta_{2}
$$


which are solutions to the problems

$$
\begin{array}{rlrl}
H_{, t}-\operatorname{div} \mathbb{T}(H, Q)= & -\lambda\left[\tilde{H} \cdot \nabla \tilde{v}_{1}+\tilde{h}_{2} \cdot \nabla \tilde{V}+\tilde{V} \cdot \nabla \tilde{h}_{1}+\tilde{v}_{2} \cdot \nabla \tilde{H}\right. & \\
& +\left(\alpha_{\theta}\left(\tilde{\theta}_{1}\right)-\alpha_{\theta}\left(\tilde{\theta}_{2}\right)\right) \tilde{\vartheta}_{1} f+\alpha_{\theta}\left(\tilde{\theta}_{2}\right) \tilde{R} f & \\
& +\left(\alpha\left(\tilde{\theta}_{1}\right)-\alpha\left(\tilde{\theta}_{2}\right)\right) g & & \text { in } \Omega^{T}, \\
& & \text { in } \Omega^{T}, \\
\operatorname{div} H=0 & & \text { on } S_{1}^{T}, \\
\bar{n} \cdot H=0, \quad \bar{n} \cdot \mathbb{D}(H) \cdot \bar{\tau}_{\alpha}=0, \quad \alpha=1,2, & & \text { on } S_{2}^{T}, \\
H_{j}=0, \quad j=1,2, \quad H_{3, x_{3}}=0 & & \text { in } \Omega, \\
\left.H\right|_{t=0}=0 & &
\end{array}
$$

and

$$
\begin{array}{ll}
R_{, t}-\varkappa \Delta R=-\lambda\left[\tilde{H} \cdot \nabla \tilde{\theta}_{1}+\tilde{h}_{2} \cdot \nabla \tilde{\mathcal{T}}+\tilde{V} \cdot \nabla \tilde{\vartheta}_{1}+\tilde{v}_{2} \cdot \nabla \tilde{R}\right] & \text { in } \Omega^{T}, \\
\bar{n} \cdot \nabla R=0 & \text { on } S_{1}^{T}, \\
R=0 & \text { on } S_{2}^{T}, \\
\left.R\right|_{t=0}=0 & \text { in } \Omega,
\end{array}
$$

where $\tilde{H}=\tilde{h}_{1}-\tilde{h}_{2}, \tilde{R}=\tilde{\vartheta}_{1}-\tilde{\vartheta}_{2}$. In view of [6] and [10, 11] we have

$$
\begin{aligned}
\|H\|_{W_{\sigma}^{2,1}\left(\Omega^{T}\right)}+\|R\|_{W_{\sigma}^{2,1}\left(\Omega^{T}\right)} & \\
\leq & c\left[\|\tilde{H}\|_{L_{\infty}\left(\Omega^{T}\right)}\left\|\nabla \tilde{v}_{1}\right\|_{L_{\eta}\left(\Omega^{T}\right)}+\left\|\tilde{h}_{2}\right\|_{L_{\infty}\left(\Omega^{T}\right)}\|\nabla \tilde{V}\|_{L_{\eta}\left(\Omega^{T}\right)}\right. \\
& \left.+\|\tilde{V}\|_{L_{\infty}\left(\Omega^{T}\right)}\left\|\nabla \tilde{h}_{1}\right\|_{L_{\xi}\left(\Omega^{T}\right)}+\left\|\tilde{v}_{2}\right\|_{L_{\infty}\left(\Omega^{T}\right)}\|\nabla \tilde{H}\|_{L_{\xi}\left(\Omega^{T}\right)}\right] \\
& +c\left(\max \left\{\left\|\tilde{\theta}_{1}\right\|_{L_{\infty}\left(\Omega^{T}\right)},\left\|\tilde{\theta}_{2}\right\|_{L_{\infty}\left(\Omega^{T}\right)}\right\}\right)\|\mathcal{T}\|_{L_{\infty}\left(\Omega^{T}\right)}\left\|\tilde{\vartheta}_{1}\right\|_{L_{\infty}\left(\Omega^{T}\right)}\|f\|_{L_{\sigma}\left(\Omega^{T}\right)} \\
& +c\left(\left\|\tilde{\theta}_{2}\right\|_{L_{\infty}\left(\Omega^{T}\right)}\|\tilde{R}\|_{L_{\infty}\left(\Omega^{T}\right)}\|f\|_{L_{\sigma}\left(\Omega^{T}\right)}\right. \\
& +a\left(\max \left\{\left\|\tilde{\theta}_{1}\right\|_{L_{\infty}\left(\Omega^{T}\right)},\left\|\tilde{\theta}_{2}\right\|_{L_{\infty}\left(\Omega^{T}\right)}\right\}\right)\|\tilde{\mathcal{T}}\|_{L_{\infty}\left(\Omega^{T}\right)}\|g\|_{L_{\sigma}\left(\Omega^{T}\right)} \\
& +\|\tilde{H}\|_{L_{\infty}\left(\Omega^{T}\right)}\left\|\nabla \tilde{\theta}_{1}\right\|_{L_{\eta}\left(\Omega^{T}\right)}+\left\|\tilde{h}_{2}\right\|_{L_{\infty}\left(\Omega^{T}\right)}\|\nabla \tilde{\mathcal{T}}\|_{L_{\eta}\left(\Omega^{T}\right)} \\
& \left.+\|\tilde{V}\|_{L_{\infty}\left(\Omega^{T}\right)}\left\|\nabla \tilde{\vartheta}_{1}\right\|_{L_{\xi}\left(\Omega^{T}\right)}+\left\|\tilde{v}_{2}\right\|_{L_{\infty}\left(\Omega^{T}\right)}\|\nabla \tilde{R}\|_{L_{\xi}\left(\Omega^{T}\right)}\right) \\
\leq & c\left(\|\tilde{V}, \tilde{\mathcal{T}}\|_{\mathcal{M}\left(\Omega^{T}\right)}\right)
\end{aligned}
$$

and from (3.8) and Lemma 3.1 we obtain $\|(V, \mathcal{T})\|_{\mathcal{M}\left(\Omega^{T}\right)} \leq c\|(\tilde{V}, \tilde{\mathcal{T}})\|_{\mathcal{M}\left(\Omega^{T}\right)}$. Hence continuity of $\phi$ follows. This concludes the proof.

Lemma 3.7. Let the assumptions of Lemmas 3.2-3.5 be satisfied. Then for every bounded subset $\mathcal{M}_{0}$ of $\mathcal{M}\left(\Omega^{T}\right)$, the family of maps

$$
\phi(\cdot, \tilde{v}, \tilde{\theta}):[0,1] \rightarrow \mathcal{M}\left(\Omega^{T}\right), \quad(\tilde{v}, \tilde{\theta}) \in \mathcal{M}_{0},
$$

is uniformly equicontinuous.

Proof. Let $(\tilde{v}, \tilde{\theta}) \in \mathcal{M}_{0}, \lambda_{i} \in[0,1], i=1,2, \lambda_{1} \geq \lambda_{2}$ and let $v_{i}, \theta_{i}$ be solutions to the problems 


$$
\begin{aligned}
& v_{i t}-\operatorname{div} \mathbb{T}\left(v_{i}, p_{i}\right)=-\lambda_{i}(\tilde{v} \cdot \nabla \tilde{v}+\alpha(\tilde{\theta}) f), \\
& \operatorname{div} v_{i}=0, \\
& \left.\bar{n} \cdot \mathbb{D}\left(v_{i}\right) \cdot \bar{\tau}\right|_{S}=0,\left.\quad \bar{n} \cdot v_{i}\right|_{S}=0, \\
& \left.v_{i}\right|_{t=0}=v_{0}, \quad i=1,2,
\end{aligned}
$$

and

$$
\begin{aligned}
& \theta_{i t}-\varkappa \Delta \theta_{i}=-\lambda_{i} \tilde{v} \cdot \nabla \tilde{\theta}, \\
& \left.\bar{n} \cdot \nabla \theta_{i}\right|_{S}=0, \\
& \left.\theta_{i}\right|_{t=0}=\theta_{0}, \quad i=1,2 .
\end{aligned}
$$

To show uniform equicontinuity we introduce the differences

$$
V=v_{1}-v_{2}, \quad P=p_{1}-p_{2}, \quad \mathcal{T}=\theta_{1}-\theta_{2}
$$

which are solutions to the problems

$$
\begin{aligned}
& V_{t}-\operatorname{div} \mathbb{T}(V, P)=-\left(\lambda_{1}-\lambda_{2}\right)(\tilde{v} \cdot \nabla \tilde{v}+\alpha(\tilde{\theta}) f), \\
& \operatorname{div} V=0, \\
& \left.\bar{n} \cdot \mathbb{D}(V) \cdot \bar{\tau}\right|_{S}=0,\left.\quad \bar{n} \cdot V\right|_{S}=0, \\
& \left.V\right|_{t=0}=0
\end{aligned}
$$

and

$$
\begin{aligned}
& \mathcal{T}_{, t}-\varkappa \Delta \mathcal{T}=-\left(\lambda_{1}-\lambda_{2}\right) \tilde{v} \cdot \nabla \tilde{\theta}, \\
& \left.\bar{n} \cdot \nabla \mathcal{T}\right|_{S}=0, \\
& \left.\mathcal{T}\right|_{t=0}=0 .
\end{aligned}
$$

In view of Lemmas 3.2-3.3,

$$
\begin{aligned}
\|V\|_{L_{\infty}\left(0, T ; W_{\eta}^{1}(\Omega)\right)}+\|\mathcal{T}\|_{L_{\infty}\left(0, T ; W_{\eta}^{1}(\Omega)\right)} \leq c\left(\left(\lambda_{1}-\lambda_{2}\right)\|(\tilde{v}, \tilde{\theta})\|_{\mathcal{M}(\Omega)}^{2}\right. \\
\left.+\left(\lambda_{1}-\lambda_{2}\right) a\left(c\|(\tilde{v}, \tilde{\theta})\|_{\mathcal{M}\left(\Omega^{T}\right)}\right)\|f\|_{L_{\sigma}\left(\Omega^{T}\right)}\right) .
\end{aligned}
$$

Let $h_{i}=v_{i, x_{3}}, \vartheta_{i}=\theta_{i, x_{3}}$. We introduce the differences

$$
H=h_{1}-h_{2}, \quad R=\vartheta_{1}-\vartheta_{2},
$$

which satisfy $H=V_{, x_{3}}, R=\mathcal{T}_{x_{3}}$. In view of Lemmas 3.4 and 3.5,

$$
\begin{aligned}
& \|H\|_{L_{\infty}\left(0, T ; W_{\xi}^{1}(\Omega)\right)}+\|R\|_{L_{\infty}\left(0, T ; W_{\xi}^{1}(\Omega)\right)} \leq c\left(\left(\lambda_{1}-\lambda_{2}\right)\|(\tilde{v}, \tilde{\theta})\|_{\mathcal{M}\left(\Omega^{T}\right)}\right. \\
& +\left(\lambda_{1}-\lambda_{2}\right) a\left(c\|(\tilde{v}, \tilde{\theta})\|_{\mathcal{M}\left(\Omega^{T}\right)}\right)\|(\tilde{v}, \tilde{\theta})\|_{\mathcal{M}\left(\Omega^{T}\right)}\|f\|_{L_{\sigma}\left(\Omega^{T}\right)} \\
& \left.+\left(\lambda_{1}-\lambda_{2}\right) a\left(c\|(\tilde{v}, \theta)\|_{\mathcal{M}\left(\Omega^{T}\right)}\right)\|g\|_{L_{\sigma}\left(\Omega^{T}\right)}\right) .
\end{aligned}
$$

From (3.9) and (3.10) the uniform equicontinuity of $\phi(\cdot, \tilde{v}, \tilde{\theta})$ follows.

Proof of Theorem 1.1. In view of the above considerations and [8, Main Theorem] the assumptions of the Leray-Schauder fixed point theorem are satisfied. Hence Theorem 1.1 is proved. 
Acknowledgements. The authors are partially supported by Polish Grant NN 201396937.

\section{References}

[1] W. Alame, On existence of solutions for the nonstationary Stokes system with slip boundary conditions, Appl. Math. (Warsaw) 32 (2005), 195-223.

[2] O. V. Besov, V. P. Il'in and S. M. Nikol'skiǔ, Integral Representations of Functions and Imbedding Theorems, Nauka, Moscow, 1975 (in Russian).

[3] P. Kacprzyk, Global regular nonstationary flow for the Navier-Stokes equations in a cylindrical pipe, Appl. Math. (Warsaw) 34 (2007), 289-307.

[4] -, Global existence for the inflow-outflow problem for the Navier-Stokes equations in a cylinder, ibid. 36 (2009), 195-212.

[5] O. A. Ladyzhenskaya, V. A. Solonnikov and N. N. Ural'tseva, Linear and Quasilinear Equations of Parabolic Type, Nauka, Moscow, 1967 (in Russian).

[6] J. Rencławowicz and W. M. Zajączkowski, Large time regular solutions to the Navier-Stokes equations in cylindrical domains, Topol. Methods Nonlinear Anal. 32 (2008), 69-87.

[7] J. Socała and W. M. Zajączkowski, Long time existence of solutions to 2d NavierStokes equations with heat convection, Appl. Math. (Warsaw) 36 (2009), 453-463.

[8] - - - Long time estimate of solutions to 3d Navier-Stokes equations coupled with heat convection, ibid. 39 (2012), 23-41.

[9] V. A. Solonnikov, A priori estimates for second order parabolic equations, Trudy Mat. Inst. Steklova 70 (1964), 133-212 (in Russian).

[10] W. M. Zajączkowski, Long time existence of regular solutions to Navier-Stokes equations in cylindrical domains under boundary slip conditions, Studia Math. 169 (2005), 243-285.

[11] - Global special regular solutions to the Navier-Stokes equations in a cylindrical domain without the axis of symmetry, Topol. Methods Nonlinear Anal. 24 (2004), 69-105.

[12] -, Global existence of axially symmetric solutions to Navier-Stokes equations with large angular component of velocity, Colloq. Math. 100 (2004), 243-263.

[13] - Global regular nonstationary flow for the Navier-Stokes equations in a cylindrical pipe, Topol. Methods Nonlinear Anal. 26 (2005), 221-286.

Jolanta Socała

State Higher Vocational School in Racibórz

Słowacki St. 55

47-400 Racibórz, Poland

E-mail: jolanta_socala@interia.pl
Wojciech M. Zajączkowski

Institute of Mathematics

Polish Academy of Sciences

Sniadeckich 8

00-956 Warszawa, Poland

E-mail: wz@impan.pl

and

Institute of Mathematics and Cryptology

Cybernetics Faculty

Military University of Technology

Kaliskiego 2

00-908 Warszawa, Poland

Received on 6.5.2010; 